\title{
INDUCIBLE DEFENSES AND TROPHIC STRUCTURE
}

\author{
Matthijs Vos, ${ }^{1,3,5}$ Antonie M. Verschoor, ${ }^{1}$ Bob W. Kooi, ${ }^{2}$ Felix L. Wäckers, ${ }^{3}$ Donald L. DeAngelis, ${ }^{4}$ \\ AND Wolf M. MooIJ ${ }^{1}$ \\ ${ }^{1}$ Department of Food Web Studies, Netherlands Institute of Ecology (NIOO-KNAW), Centre for Limnology, \\ Rijksstraatweg 6, 3631 AC Nieuwersluis, The Netherlands \\ ${ }^{2}$ Department of Theoretical Biology, Faculty of Earth and Life Sciences, Vrije Universiteit, De Boelelaan 1087, $1081 \mathrm{HV}$, \\ Amsterdam, The Netherlands \\ ${ }^{3}$ Department of Multitrophic Interactions, Netherlands Institute of Ecology (NIOO-KNAW), Centre for Terrestrial Ecology, \\ Boterhoeksestraat 48, 6666 GA Heteren, The Netherlands \\ ${ }^{4}$ U.S. Geological Survey, Biological Resources Division and University of Miami, Department of Biology, P.O. Box 249118, \\ Coral Gables, Florida 33124, USA
}

\begin{abstract}
Resource edibility is a crucial factor in ecological theory on the relative importance of bottom-up and top-down control. Current theory explains trophic structure in terms of the relative abundance and succession of edible and inedible species across gradients of primary productivity. We argue that this explanation is incomplete owing to its focus on inedibility and the assumption that plants and herbivores have fixed defense levels. Consumer-induced defenses are an important source of variation in the vulnerability of prey and are prevalent in natural communities. Such induced defenses decrease per capita consumption rates of consumers but hardly ever result in complete inedibility. When defenses are inducible a prey population may consist of both undefended and defended individuals. Here we use food chain models with realistic parameter values to show that variation in consumption rates on different prey types causes a gradual instead of stepwise increase in the biomass of all trophic levels in response to enrichment. Such all-level responses have been observed in both aquatic and terrestrial ecosystems and in microbial food chains in the laboratory. We stress that, in addition to the known food web effects of interspecific variation in edibility, intraspecific variation in edibility is another form of within-trophic-level heterogeneity that also has such effects. We conclude that inducible defenses increase the relative importance of bottom-up control.
\end{abstract}

Key words: biomass responses; bottom-up control; edibility; enrichment; food chain; food web; infochemicals; multitrophic interactions; primary productivity; top-down control.

\section{INTRODUCTION}

Understanding the structure of complex natural food webs remains a major task in ecology. Debates on trophic structure have revolved around simple food chain models that predict the distribution of biomass over trophic levels in response to enrichment. The basic model was conceived by Rosenzweig and MacArthur (1963) and further formalized, developed, and analyzed by Rosenzweig (1971, 1973), Oksanen et al. (1981), Abrams (1993), Kretzschmar et al. (1993), Abrams and Roth (1994), and Oksanen and Oksanen (2000). The predictions of the Oksanen et al. (1981) model have become well known as the hypothesis of exploitation ecosystems (EEH) and involve a stepwise increase in the biomass of trophic levels under enrichment (Oksanen et al. 1981, Leibold 1989, Oksanen and Oksanen 2000). For bitrophic systems in stable equilibrum this model predicts that plant biomass is exclusively controlled by herbivores and will not respond to enrichment. Similarly, equilibrium herbivore biomass is exclusively controlled by carnivores in tritrophic systems

Manuscript received 9 October 2003; revised and accepted 1 April 2004. Corresponding Editor (ad hoc): K. S. McCann.

${ }^{5}$ E-mail: m.vos@nioo.knaw.nl and should not respond to increases in primary productivity. This last prediction was supported by an experimental study within a natural river (Wootton and Power 1993).

However, a number of field studies in both aquatic and terrestrial systems did not support EEH predictions. Plant biomass has been shown to increase with productivity, irrespective of the number of trophic levels (Hansson 1992, Brett and Goldman 1997, Chase et al. 2000, Oksanen and Oksanen 2000: Fig. 3). Biomasses of plants and herbivores have been shown to increase in concert in response to elevated nutrient levels (Akcakaya et al. 1995, Leibold 1996, Brett and Goldman 1997) and annual precipitation, a good measure of resource supply rate in terrestrial systems (Rosenzweig 1968, Milchunas and Lauenroth 1993, Chase et al. 2000). Even a laboratory study of bitrophic and tritrophic microbial food chains, which was designed to minimize confounding factors such as omnivory or the presence of inedible species, showed that productivity increased the abundances of all trophic levels (Kaunzinger and Morin 1998).

Such discrepancies between EEH predictions and the outcome of both laboratory and field studies requires the identification of ecological factors not present in 
the Oksanen et al. (1981) model that govern trophiclevel biomass responses in nature. Variation in the edibility of different plant species may be a good starting point, as theory predicts such heterogeneity to have important community-level consequences (Leibold 1989, Power 1992, Grover 1995, Leibold et al. 1997, Oksanen and Oksanen 2000, Agrawal 2001, Steiner 2001) and to change biomass responses to enrichment (Leibold 1989, Abrams 1993, Leibold 1996).

Inducible defenses constitute another important source of heterogeneity in edibility in natural communities. Their effect on trophic structure has hardly been studied. This is surprising, as inducible defenses have been documented for a wide variety of species in many different ecosystems. In terrestrial plants they involve herbivore-induced production of spines (Gowda 1996), thorns (Gomez and Zamora 2002), toxins, and synomones (Karban and Baldwin 1997). In aquatic systems, herbivores may induce colony formation in freshwater algae (Hessen and Van Donk 1993, Lampert et al. 1994, Van Donk et al. 1999) and marine algae (Tang 2003). Most induced defenses will affect handling times and/or attack rates of consumers and thus their functional responses (Jeschke and Tollrian 2000). Inducible defenses in animals include refuge use, reduced activity, adaptive life history changes, and the formation of helmets or spines. They occur in freshwater zooplankton (protozoa, rotifers, cladocera; Havel 1987, De Meester et al. 1999, Gilbert 1999, Kuhlmann et al. 1999, Tollrian and Dodson 1999, Vos et al. 2002), snails (Turner et al. 2000), insects (Dahl and Peckarsky 2002), amphibia (Anholt and Werner 1999), fish (Brönmark et al. 1999), marine colonial invertebrates (bryozoa and gorgonacea, Harvell 1999) and barnacles (Lively 1999).

The goal of this paper is to investigate how inducible defenses affect the distribution of biomass over trophic levels when the system is enriched. We focus on three aspects of inducible defenses that have been shown to be important in a variety of empirical studies, but that have not been investigated in concert in theoretical studies: (1) The induction of defenses depends on consumer density (e.g., Anholt and Werner 1999, Kuhlmann et al. 1999, Van Donk et al. 1999), (2) both undefended prey and prey with induced defenses may be present at a given moment, over a range of consumer densities (Hessen and Van Donk 1993, Lampert et al. 1994), and (3) defended prey are not invulnerable (Jeschke and Tollrian 2000).

In the current paper we incorporate these aspects into a classical food chain model (Oksanen et al. 1981, also see Kretzschmar et al. 1993). We study this model analytically and parameterize it for a well-studied system to investigate the effects of inducible defenses in an ecologically relevant domain. This provides results that are amenable to empirical testing.

In this respect a planktonic food chain consisting of algae, herbivorous rotifers, and carnivorous rotifers is a good model system. For example, strains of Scenedesmus spp. algae differ in edibility to Brachionus spp. rotifers, owing to inducible colony formation (Lürling 1999). Different Brachionus spp. rotifers vary in edibility to consumers like Asplanchna spp. rotifers, owing to inducible spine formation and size differences (Gilbert 1999). Colonies and spines effectively lower the maximum ingestion rates achieved by gape-limited consumers. Of the above-mentioned organisms, different strains or species exist that (1) always have the undefended phenotype, (2) have inducible defenses, or (3) are permanently defended. Inducible defenses cause intraspecific heterogeneity in both the plant (Hessen and Van Donk 1993, Lampert et al. 1994) and the herbivore population (Gilbert 1967).

Using models of bitrophic and tritrophic food chains, we specifically address the following question: Does an increase in primary productivity cause gradual biomass increases of adjacent trophic levels, when inducible defenses are incorporated in the classical Oksanen et al. (1981) food chain model?

\section{Methods \\ Model structure}

We use a classical food chain model (Oksanen et al. (1981), with a slight modification to include nonherbivory mortality in the plants, as the basis for the three scenarios where defenses are absent, permanent, or inducible. Our standard food chain model is defined by

$$
\begin{aligned}
\frac{d P_{i}}{d t} & =P_{i}\left[r_{i}\left(1-\frac{P_{i}}{k}\right)-\frac{v_{i 1} H_{j}}{1+v_{i 1} h_{i 1} P_{i}}-s_{i 1}\right] \\
& =\text { logistic plant growth }- \text { herbivory }- \text { mortality }
\end{aligned}
$$

$$
\begin{aligned}
\frac{d H_{j}}{d t} & =H_{j}\left(\frac{c_{i 1} v_{i 1} P_{i}}{1+v_{i 1} h_{i 1} P_{i}}-\frac{v_{j 2} C}{1+v_{j 2} h_{j 2} H_{j}}-s_{j 2}\right) \\
& =\text { herbivore growth }- \text { carnivory }- \text { mortality }
\end{aligned}
$$

$$
\begin{aligned}
\frac{d C}{d t} & =C\left(\frac{c_{2} v_{j 2} H_{j}}{1+v_{j 2} h_{j 2} H_{j}}-s_{3}\right) \\
& =\text { carnivore growth }- \text { mortality }
\end{aligned}
$$

where subscripts $i$ and $j$ equal 1 in the undefended state and 2 in the defended state, $t$ is time, $r_{i}$ intrinsic growth rate, and $k$ the carrying capacity of plants (a measure for primary productivity), $s$ the death rate (with subscripts $i$ and $j$ denoting the "state of defense" and 1, 2 , or 3 the trophic level), $c_{i 1}$ the efficiency of plant conversion into herbivore biomass, and $c_{2}$ the herbivore conversion efficiency. The trophic interaction parameters are: $v_{i 1}$, herbivore search rate; $v_{j 2}$, carnivore search rate; $h_{i 1}$, herbivore handling time; and $h_{j 2}$, carnivore handling time. These parameters may be different for defended and undefended prey, but are independent of 
the defense level of their consumers. All consumption rates follow Holling type II functional responses. Herbivores have longer handling times $\left(h_{21}\right)$ on defended plants $\left(h_{21}>h_{11}\right)$. This decreases their maximum ingestion rate $\left(1 / h_{21}<1 / h_{11}\right)$. Attack rates may be decreased on defended plants. The same holds for carnivores feeding on herbivores. By definition, a food chain without defenses is composed of only $P_{1}, H_{1}$, and $C$, while only $P_{2}, H_{2}$, and $C$ exist in a food chain with permanent defenses. Our analysis focuses on three scenarios: no defenses, permanent defenses, and inducible defenses, in both plants and herbivores. This choice provides a clear contrast and demarcation, but ignores the possible permutations of these scenarios.

We incorporate inducible defenses in the above system by allowing both an undefended and a defended part in each prey population. Induction and decay of defenses cause consumer density-dependent flows between these parts. Induction is minimal at low consumer densities and maximal at high consumer densities. The reverse is true for the decay of defenses. The following set of differential equations defines the rates of change of undefended and defended plants, undefended and defended herbivores, and carnivores:

$$
\begin{aligned}
& \frac{d P_{1}}{d t}=P_{1}\left[r_{1}\left(1-\frac{P_{1}}{k}-\frac{P_{2}}{k}\right)\right. \\
& \left.-\frac{v_{11}\left(H_{1}+H_{2}\right)}{1+v_{11} h_{11} P_{1}+v_{21} h_{21} P_{2}}-s_{11}\right] \\
& \text { - } P_{1} I_{1}\left(H_{1}, H_{2}\right)+P_{2} D_{1}\left(H_{1}, H_{2}\right) \\
& \frac{d P_{2}}{d t}=P_{2}\left[r_{2}\left(1-\frac{P_{2}}{k}-\frac{P_{1}}{k}\right)\right. \\
& \left.-\frac{v_{21}\left(H_{1}+H_{2}\right)}{1+v_{11} h_{11} P_{1}+v_{21} h_{21} P_{21}}-s_{21}\right] \\
& +P_{1} I_{1}\left(H_{1}, H_{2}\right)-P_{2} D_{1}\left(H_{1}, H_{2}\right) \\
& \frac{d H_{1}}{d t}=H_{1}\left(\frac{c_{11} v_{11} P_{1}+c_{21} v_{21} P_{2}}{1+v_{11} h_{11} P_{1}+v_{21} h_{21} P_{1}}\right. \\
& \left.-\frac{v_{12} C}{1+v_{12} h_{12} H_{1}+v_{22} h_{22} H_{2}}-s_{12}\right) \\
& -H_{1} I_{2}(C)+H_{2} D_{2}(C) \\
& \frac{d H_{2}}{d t}=H_{2}\left(\frac{c_{11} v_{11} P_{1}+c_{21} v_{21} P_{2}}{1+v_{11} h_{11} P_{1}+v_{21} h_{21} P_{2}}\right. \\
& \left.-\frac{v_{22} C}{1+v_{12} h_{12} H_{1}+v_{22} h_{22} H_{2}}-s_{22}\right) \\
& +H_{1} I_{2}(C)-H_{2} D_{2}(C) \\
& \frac{d C}{d t}=C\left[\frac{c_{2}\left(v_{12} H_{1}+v_{22} H_{2}\right)}{1+v_{12} h_{12} H_{1}+v_{22} h_{22} H_{2}}-s_{3}\right]
\end{aligned}
$$

with herbivores and carnivores consuming two re- source types. The model formulation used here is slightly modified after Kretzschmar et al. (1993). The induction rate of defenses, $I$, and the decay rate of defenses, $D$, are functions of the total consumer density. Note that induction is a process that subtracts from the undefended part of the resource population and adds to the defended part. Decay of defenses takes away from the defended part of a prey population and adds to the undefended part. Induction and decay rates are

$$
\begin{aligned}
I_{1}\left(H_{1}, H_{2}\right) & =i_{1}\left\{1-\left[1+\left(\frac{H_{1}+H_{2}}{g_{1}}\right)^{b_{1}}\right]^{-1}\right\} \\
& =\text { induction rate of plant defenses } \\
D_{1}\left(H_{1}, H_{2}\right) & =i_{1}\left[1+\left(\frac{H_{1}+H_{2}}{g_{1}}\right)^{b_{1}}\right]^{-1} \\
& =\text { decay rate of plant defenses } \\
I_{2}(C) & =i_{2}\left\{1-\left[1+\left(\frac{C}{g_{2}}\right)^{b_{2}}\right]^{-1}\right\} \\
& =\text { induction rate of herbivore defenses } \\
D_{2}(C) & =i_{2}\left[1+\left(\frac{C}{g_{2}}\right)^{b_{2}}\right]^{-1} \\
& =\text { decay rate of herbivore defenses }
\end{aligned}
$$

where $g_{1}$ is the density of herbivores at which plant defense induction reaches half its maximum rate and $b_{1}$ a shape parameter of the plant defense induction and decay functions. These parameters are $g_{2}$ and $b_{2}$ for herbivore defenses, with half-saturation parameter $g_{2}$ now denoting carnivore density. This system (Eqs. 3ad) ensures that eventually all plants and/or herbivores become undefended when their consumers are absent. In their presence, a balance of induction and decay will be approached at a rate that depends on parameter $i$. Summation of Eqs. $2 \mathrm{a}$ and $\mathrm{b}$ shows that total plant biomass does not change due to the induction process. This also holds for total herbivore biomass.

The bitrophic system is obtained by using only Eqs. 2a-c for $P_{1}, P_{2}$, and $H_{1}$, and setting $C$ and $H_{2}$ in these equations to zero. State variables are further explained in Table 1.

\section{Model parameterization}

For those results that depend on numerical analysis we parameterized the model using values from the literature and our own experimental data (A. M. Verschoor, unpublished data). All parameters and literature sources are listed in Table 2. In this planktonic model system no costs of inducible defenses in terms of reduced growth rates can be detected (Gilbert 1999, Lürling and Van Donk 2000). However, colony formation in algae leads to increased sedimentation rates, which we incorporated as an increased mortality rate $s_{21}$. Note that the default set of parameter values in Table 2 has defenses increasing handling times, thus decreasing 
TABLE 1. List of state variables used in the model, their units, and interpretation.

\begin{tabular}{cll}
\hline \hline Variable & Unit & \multicolumn{1}{c}{ Interpretation } \\
\hline$P_{1}$ & $\mathrm{mg} \mathrm{C} / \mathrm{L}$ & undefended plants (algae) \\
$P_{2}$ & $\mathrm{mg} \mathrm{C/L}$ & defended plants (algae) \\
$H_{1}$ & $\mathrm{mg} \mathrm{C/L}$ & undefended herbivores (zooplankton) \\
$H_{2}$ & $\mathrm{mg} \mathrm{C/L}$ & defended herbivores (zooplankton) \\
$\mathrm{C}$ & $\mathrm{mg} \mathrm{C} / \mathrm{L}$ & carnivores (zooplankton) \\
\hline
\end{tabular}

maximum ingestion rates, without an effect on attack rates. However, in many systems defenses will reduce attack rates and this possibility is included in the sensitivity analysis. Theory predicts trade-offs to be important in systems with inducible defenses and therefore we also include alternative trade-off scenarios in the sensitivity analysis.

\section{Model analysis}

We started by obtaining analytical results on trophic level abundances for the different defense scenarios, depending on the carrying capacity $k$. Since the discrepancy between EEH predictions and actual data centers around the plant level in bitrophic systems and the herbivore level in tritrophic systems, these were our focal levels. The analytical results are general and not concerned with any particular system.

The next step was a numerical analysis of a planktonic model system, where Eqs. 1 and 2 represented algae and rotifers. We obtained biomass distributions over trophic levels in bitrophic and tritrophic food chains, for a range of carrying capacities $(k)$. This range allows one, two, or three trophic levels to exist, and includes both stable equilibria and oscillatory periodic behavior. We concluded this analysis by checking the sensitivity of biomass responses in the bitrophic inducible defense scenario to changes in some of the assumptions, model components, and parameter values used. This sensitivity analysis widens the scope of the numerical analysis, providing a link between general analytical results and specific results for the planktonic example. We investigated the sensitivity of the model to the following changes: (1) changing the herbivores' response to plant defense from purely an effect on handling times $\left(h_{21}\right)$ to an effect on attack rates $\left(v_{21}\right)$ as well; (2) allowing plant defenses to reduce herbivore attack rates $\left(v_{21}\right)$ without an increase in handling times $\left(h_{21}\right)$; (3) allowing quantitatively weaker and stronger

TABLE 2. Definitions of model variables and parameters, and their default numerical values. Numbers refer to the sources on which the parameter estimates were based (references are at the bottom of the table).

\begin{tabular}{|c|c|c|c|c|}
\hline Parameter & Value & $\begin{array}{l}\text { References } \\
\text { (see } \\
\text { footnotes) }\end{array}$ & Units & Interpretation \\
\hline$b_{1}$ & 2.05 & $1,2,3$ & & shape of plant defense functions (induction/decay) \\
\hline$b_{2}$ & 1.5 & $2,4,5$ & & shape of herbivore defense functions (induction/decay) \\
\hline$c_{11}$ & 0.36 & 6 & $\mathrm{mg} \mathrm{C} / \mathrm{mg} \mathrm{C}$ & conversion efficiency of undefended plant-herbivore \\
\hline$c_{21}$ & 0.36 & 6 & $\mathrm{mg} \mathrm{C} / \mathrm{mg} \mathrm{C}$ & conversion efficiency of defended plant-herbivore \\
\hline$c_{2}$ & 0.5 & 7 & $\mathrm{mg} \mathrm{C} / \mathrm{mg} \mathrm{C}$ & conversion efficiency of herbivore-carnivore \\
\hline$g_{1}$ & 0.06 & $1,2,3$ & $\mathrm{mg} \mathrm{C} / \mathrm{L}$ & half-saturation value for induction/decay of plant defenses \\
\hline$g_{2}$ & 0.020 & $2,4,5$ & $\mathrm{mg} \mathrm{C} / \mathrm{L}$ & half-saturation value for induction/decay of herbivore defenses \\
\hline$i_{1}$ & 1.0 & 11 & $\mathrm{~d}^{-1}$ & scaling parameter for induction/decay rate of plant defenses \\
\hline$i_{2}$ & 1.0 & 11 & $\mathrm{~d}^{-1}$ & scaling parameter for induction/decay rate of herbivore defenses \\
\hline$k$ & free & & $\mathrm{mg} \mathrm{C} / \mathrm{L}$ & carrying capacity for defended and undefended plants \\
\hline$r_{1}$ & 1.42 & 8 & $\mathrm{~d}^{-1}$ & intrinsic rate of increase for undefended plants \\
\hline$r_{2}$ & 1.42 & 8 & $d^{-1}$ & intrinsic rate of increase for defended plants \\
\hline$s_{11}$ & 0.145 & 9 & $\mathrm{~d}^{-1}$ & natural death rate for undefended plants (sedimentation) \\
\hline$s_{21}$ & 0.18 & 9 & $\mathrm{~d}^{-1}$ & natural death rate for defended plants (sedimentation) \\
\hline$s_{12}$ & 0.17 & 10 & $d^{-1}$ & natural death rate for undefended herbivores \\
\hline$s_{22}$ & 0.17 & 10 & $\mathrm{~d}^{-1}$ & natural death rate for defended herbivores \\
\hline$s_{3}$ & 0.125 & 5 & $\mathrm{~d}^{-1}$ & natural death rate of consumers \\
\hline$h_{11}$ & 0.5 & 11 & $\mathrm{~d} \cdot \mathrm{mg} \mathrm{C} \mathrm{C}^{-1} \cdot \mathrm{mg} \mathrm{C}^{-1}$ & handling time of herbivores on undefended plants \\
\hline$h_{21}$ & 1.04 & 11 & $\mathrm{~d} \cdot \mathrm{mg} \mathrm{C}^{-1} \cdot \mathrm{mg} \mathrm{C}^{-1}$ & handling time of herbivores on defended plants \\
\hline$h_{12}$ & 0.83 & $7,12,13$ & $\mathrm{~d} \cdot \mathrm{mg} \mathrm{C} \mathrm{C}^{-1} \cdot \mathrm{mg} \mathrm{C}^{-1}$ & handling time of consumers on undefended herbivores \\
\hline$h_{22}$ & 3.33 & $7,12,13$ & $\mathrm{~d} \cdot \mathrm{mg} \mathrm{C}-1 \cdot \mathrm{mg} \mathrm{C}^{-1}$ & handling time of consumers on defended herbivores \\
\hline$v_{11}$ & 0.77 & 11 & $\mathrm{~L} \cdot \mathrm{d}^{-1} \cdot \mathrm{mg} \mathrm{C}^{-1}$ & herbivore search rate on undefended plants \\
\hline$v_{21}$ & 0.77 & 11 & $\mathrm{~L} \cdot \mathrm{d}^{-1} \cdot \mathrm{mg} \mathrm{C}^{-1}$ & herbivore search rate on defended plants \\
\hline$v_{12}$ & 2.71 & 12,13 & $\mathrm{~L} \cdot \mathrm{d}^{-1} \cdot \mathrm{mg} \mathrm{C} \mathrm{C}^{-1}$ & carnivore search rate on undefended herbivores \\
\hline$v_{22}$ & 2.71 & 12,13 & $\mathrm{~L} \cdot \mathrm{d}^{-1} \cdot \mathrm{mg} \mathrm{C} \mathrm{C}^{-1}$ & carnivore search rate on defended herbivores \\
\hline
\end{tabular}

Sources: 1, Lürling (1999); 2, Dumont et al. (1975); 3, Jørgensen et al. (1991); 4, Gilbert and Waage (1967); 5, Gilbert (1976); 6, Walz (1993); 7, Nandini and Sarma (1999); 8, Lürling and Van Donk (2000); 9, Laroque et al. (1996); 10, Stemberger (1990); 11, A.M. Verschoor (unpublished data); 12, Iyer and Rao (1996); 13, Gilbert (1967).

Note: The Methods section explains the sensitivity analysis. 


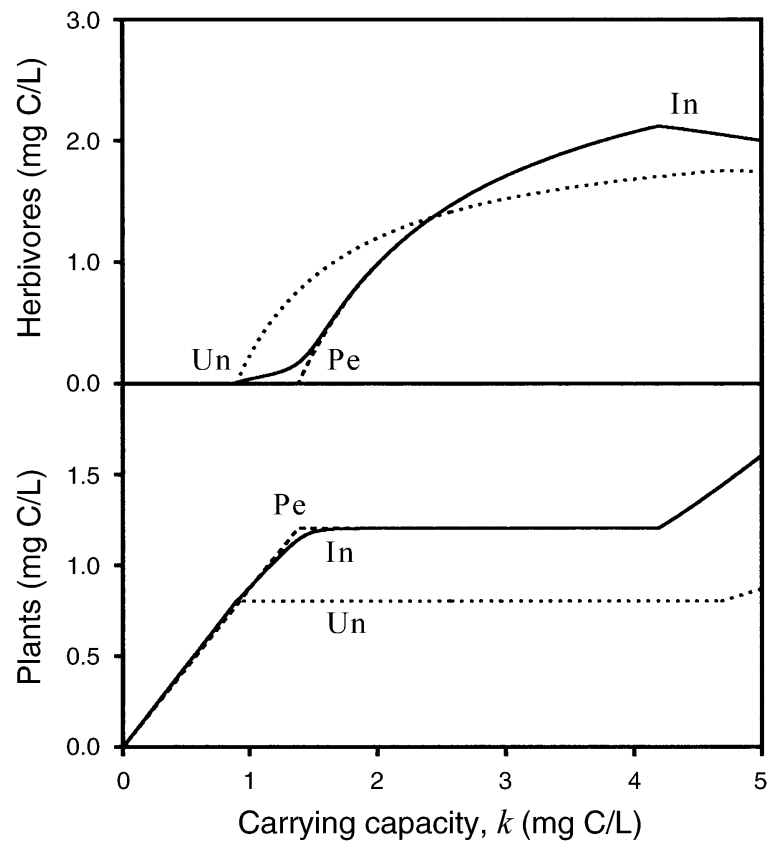

FIG. 1. Equilibria of the bitrophic food chain model when plant defenses are absent (Un, dotted lines), permanent (Pe, dashed lines), or inducible (In, solid lines) along a productivity gradient. The average biomass of herbivores (top graph) and plants (bottom graph) is shown on the ordinate axis. Parameter values are listed in Table 2.

effects on handling times $\left(h_{21}\right)$ than estimated for this model system; (4) using a linear instead of Holling type II herbivore functional response $\left(h_{11}=h_{21}=0\right)$, with defenses causing decreased attack rates $\left(v_{21}\right) ;(5)$ allowing plant defenses to decrease the conversion efficiency $\left(c_{21}\right)$ to herbivore biomass; (6) incorporating induced plants as inedible $\left(v_{21}=0\right)$; (7) including no cost for plant defenses $\left(s_{11}=s_{21}\right)$; (8) including plant defense costs as a reduced intrinsic growth rate $r_{2}$ for induced plants instead of an increased mortality rate; (9) simultaneously increasing or decreasing the values of the defense induction and decay parameters $b_{1}$ and $g_{1}$; and (10) evaluating the results for a range of the defense induction and decay parameter $i_{1}$. All increases and decreases amounted to $5-30 \%$ of the estimated parameter values (see Table 2), and were implemented in steps of $5 \%$.

In addition we compared the bitrophic inducible defense scenario with a bitrophic scenario that has two species at the plant level. These plant species have different but fixed defense levels. In this multiprey model the defense induction and decay terms of Eqs. $2 \mathrm{a}, \mathrm{b}$ are lacking. We will refer to these models as, respectively, the intraspecific heterogeneity model (with inducible defenses), and the interspecific heterogeneity model (with fixed, but different defenses). In this comparison we focus on cases where $v_{21}<v_{11}$. We allow $h_{21}$ to be $\geq h_{11}$ and defended plants may be inedible $\left(v_{21}=0\right)$. The comparison is made for a pro- ductivity gradient that allows an equilibrium of plants and herbivores in both the intraspecific and interspecific heterogeneity model and allows an increase in the fraction of defended plants in the inducible defense model ( $k=0.95-1.25 \mathrm{mg} \mathrm{C} / \mathrm{L}$, see Numerical results). This analysis facilitates a comparison with earlier work (Abrams 1993, Kretzschmar et al. 1993, Grover 1995, Leibold 1996, Bohannan and Lenski 1999).

\section{Results \\ Analytical results}

Biomass accrues in the classical stepwise fashion in the bitrophic food chain scenario without plant defenses (e.g., Fig. 1). At very low carrying capacities only plants exist. From Eq. 1a it follows that the equilibrium value $P_{1}^{*}$ increases linearly with increases in $k$ :

$$
P_{1}^{*}=\left(\frac{r_{1}-s_{11}}{r_{1}}\right) k
$$

At a higher level of primary productivity a point will be reached that allows herbivores to exist. The carrying capacity $k_{\mathrm{tc}}$ at this transcritical bifurcation point can be derived by allowing the hump-shaped plant isocline to intersect the vertical herbivore isocline at $H=0$ :

$$
k_{\mathrm{tc}}=\left(\frac{r_{1}}{r_{1}-s_{11}}\right)\left[\frac{s_{12}}{v_{11}\left(c_{11}-s_{12} h_{11}\right)}\right] .
$$

Above this carrying capacity $k_{\mathrm{tc}}$ the equilibrium plant biomass $P_{1}^{*}$ is

$$
P_{1}^{*}=\frac{s_{12}}{v_{11}\left(c_{11}-h_{11} s_{12}\right)}
$$

which is independent of the carrying capacity $k$, as predicted by EEH theory. Equilibrium herbivore biomass increases with further increases in productivity:

$$
H^{*}=\frac{c_{11} P_{1}^{*}}{s_{12}}\left[r_{1}\left(1-\frac{P_{1}^{*}}{k}\right)-s_{11}\right]
$$

as the first factor on the right side of Eq. 7 is positive and the second factor an increasing function of $k$ that is positive above $k_{\mathrm{tc}}$. Under further enrichment the system will remain stable until the Hopf bifurcation is crossed at the carrying capacity $k_{\mathrm{Hopf}}$,

$$
k_{\mathrm{Hopf}}=\left(\frac{r}{r_{1}-s_{11}}\right)\left[\frac{2 s_{12}}{v_{11}\left(c_{11}-s_{12} h_{11}\right)}+\frac{1}{v_{11} h_{11}}\right]
$$

(also see Rosenzweig 1971, Fussmann et al. 2000).

In the case of permanent plant defenses, herbivores require a higher carrying capacity to invade when plants are defended. In the food chain scenario with inducible defenses, herbivores are also not able to exist at a low carrying capacity. In the absence of herbivores, plant defenses are not induced and the results are equal to the undefended scenario (Eq. 4, also see the example in Fig. 1). At carrying capacities above $k_{\mathrm{tc}}$ induction will cause a certain fraction, $x=P_{2} /\left(P_{1}+P_{2}\right)$, of the plant population to be in the defended state. Setting 
the right side of Eq. 2c (bitrophic case) to zero shows that at equilibrium total plant biomass $P_{\text {tot }}(x)$ (being $P_{1}$ $\left.+P_{2}\right)$, must fulfill the following relationship:

$$
\begin{gathered}
P_{\text {tot } 1}(x)=s_{12} /\left[c_{1} v_{11}+\left(c_{1} v_{21}-c_{1} v_{11}\right) x-s_{12} v_{11} h_{11}\right. \\
\left.-s_{12}\left(v_{21} h_{21}-v_{11} h_{11}\right) x\right]
\end{gathered}
$$

where we have assumed $c_{1}=c_{11}=c_{21}$. Eq. 9 shows that an increase in the fraction of defended plants $x$ will cause the entire plant level to increase for $s_{12}\left(v_{21} h_{21}\right.$ $\left.-v_{11} h_{11}\right)>c_{1}\left(v_{21}-v_{11}\right)$. When defenses increase handling times and thus maximum ingestion rates, but do not affect attack rates $\left(h_{21}>h_{11}, v_{21}=v_{11}\right)$, Eq. 9 reduces to

$$
P_{\text {tot } 1}(x)=\frac{s_{12}}{c_{1} v_{11}-s_{12} v_{11} h_{11}-s_{12} v_{11}\left(h_{21}-h_{11}\right) x} .
$$

In this case any increase in the fraction of defended plants $x$ will decrease the denominator at the right side of Eq. 10 , leading to an increase in the total plant biomass.

It is possible to demonstrate analytically for the case $v_{21}=v_{11}$ and $r_{1}=r_{2}$, at least, that the equilibrium point, $P_{\text {tot }}$, for the bitrophic inducible defense case is an increasing function of plant carrying capacity. To show this we return to Eqs. 2a,b,c, setting $H_{1}=H, H_{2}=0$, and $C=0$. By combining Eqs. 2a,b we find a relationship between $H$ and $x$ in equilibrium

$$
H(x)=g\left\{\frac{1}{1-\left[\left(\frac{s_{21}-s_{11}}{i}\right) x(1-x)+x\right]}-1\right\}^{1 / b} .
$$

By combining Eqs. 2a,b,c we find an expression for the carrying capacity $k$, necessary to keep the system at a given equilibrium of $x$ :

$$
k=\frac{r P_{\text {tot }}(x)}{r-\left[s_{11}(1-x)+s_{21} x\right]-\left(\frac{s_{21}}{c_{1}}\right)\left[\frac{H(x)}{P_{\text {tot }}(x)}\right]}
$$

where $r=r_{1}=r_{2}$. By entering a range of values for $x^{*}$ in Eq. 12 one obtains a view of the range of carrying capacities for which the fraction of defended plants increases. In order to prove a positive relation between $P_{\text {tot }}$ and $k$ we have rewritten Eq. 12 as an equation for $P_{\text {tot }}$ and obtain a function of $x$ and $k$, now called $P_{\text {tot }}$ ${ }_{2}(x, k)$ to distinguish it from $P_{\text {tot } 1}(x)$ :

$$
\begin{aligned}
P_{\text {tot } 2}(x, k)= & \frac{1}{2} \frac{k}{r}\left[r-\left(s_{11}(1-x)+s_{21} x\right)\right] \\
\pm & {\left[\left(\frac{1}{2} \frac{k}{r}\left[r-\left(s_{11}(1-x)+s_{21} x\right)\right]\right)^{2}\right.} \\
& \left.-\frac{k}{r} \frac{s_{12}}{c_{1}} H(x)\right]^{1 / 2} .
\end{aligned}
$$

Hence, we have three relationships between $P_{\text {tot }}, x$, and $H$, namely, Eqs. 10, 11, and 13, and the equilibrium

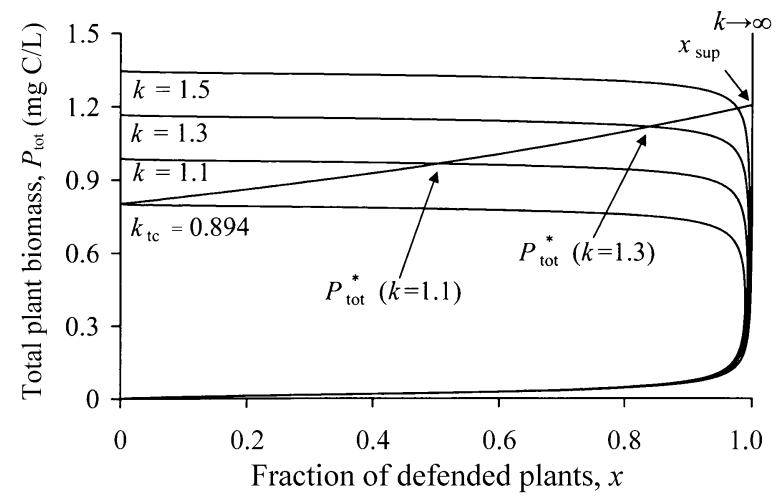

FIG. 2. Total plant biomass $P_{\text {tot } 1}$, as an increasing function of $x$ (see Eq. 10), and a family of independently derived curves for $P_{\text {tot } 2}$ as a function of $x$ and $k$ (see Eq. 13), plotted for different values of the carrying capacity, $k$. The intersection point of these curves fixes the total equilibrium plant biomass. This intersection point occurs at higher values of $P_{\text {tot }}$ as the carrying capacity $k$ increases. An increase in $k$ from $k=1.1$ to $k=1.3$ causes a shift from the curve $P_{\text {tot } 2}(x, k=$ $1.1)$ to $P_{\text {tot } 2}(x, k=1.3)$ and a shift of the equilibrium, namely, where these curves intersect with $P_{\text {tot } 1}(x)$.

values $P_{\mathrm{tot}}^{*}, x^{*}$, and $H^{*}$ satisfy all three relationships simultaneously. When $H(x)$ given by Eq. 11 is substituted into Eq. 13, only two relationships Eqs. 10 and 13 remain for $P_{\mathrm{tot}}^{*}$ and $x^{*}$. The equilibrium value $P_{\mathrm{tot}}^{*}$ is given by the intersection between $P_{\text {tot } 1}(x)$ of Eq. 10 and $P_{\text {tot } 2}(x, k)$ of Eq. 13 where $k$ is the free parameter.

The positive and negative roots or branches of Eq. 13 form a hump-shaped curve when plotted together in the $\left(x, P_{\text {tot }}\right)$-plane (Fig. 2). For a given value of $k$ this hump-shaped curve completely encompasses the hump-shaped curve for a lower value of $k$, for all permissible values of $x$. Thus the positive branches, $P_{\text {tot } 2}^{+}(x, k)$, of Eq. 13 for higher $k$ values always lie above those for lower $k$ values for the entire permissible range of $x$, $\left(0 \leq x<x_{\text {sup }}<1\right)$, where $x_{\text {sup }}$ is the supremum value of $x$ that occurs for $k>0$. Analogously, the negative branches, $P_{\text {tot } 2}^{-}(x, k)$, for higher $k$ values have to lie below those for lower $k$ values for all permissible values of $x$. Mathematically this means that for the positive branch the partial derivative with respect to $k$ is positive, for all $x$, while it is negative for the negative branch, as demonstrated in the Appendix. These results and the fact that $P_{\text {tot } 1}(x)$ is an increasing function of $x$ ensure that the intersection between $P_{\text {tot } 1}(x)$ and $P_{\text {tot } 2}(x, k)$ occurs for a higher value of $P_{\text {tot }}$ when the carrying capacity $k$ is increased, both for the positive and negative branches of $P_{\text {tot } 2}(x, k)$. This means that, under our assumptions, inducible defenses will cause the plant level to increase with an increase in productivity. This is in contrast with the classical EEH prediction that plant biomass in a bitrophic system remains constant under enrichment.

We now move on to the tritrophic system with inducible defenses. The analytical results for the herbi- 


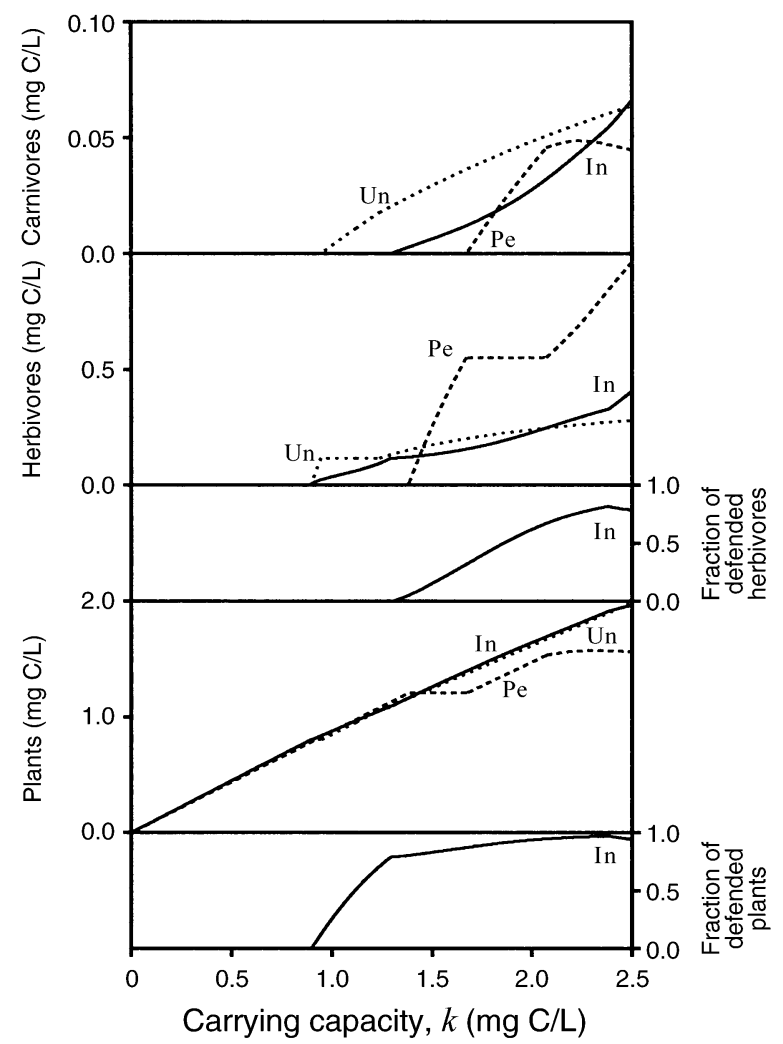

FIG. 3. Biomass responses of a tritrophic food chain when defenses are absent (Un, dotted lines), permanent (Pe, dashed lines), or inducible (In, solid lines), along a productivity gradient. From top to bottom: the average biomass of carnivores $C$, herbivores $H_{1}+H_{2}$, the fraction of defended herbivores $y=H_{2} /\left(H_{1}+H_{2}\right)$, average plant biomass $P$, and the fraction of defended plants $x=P_{2} /\left(P_{1}+P_{2}\right)$. Parameter values are listed in Table 2. Population densities oscillate at high values of the carrying capacity $k$.

vore level in this case are similar to those for the plant level in the bitrophic case. Total herbivore biomass $H_{\text {tot }}$ (being $H_{1}+H_{2}$ ) at equilibrium must fulfill the following relationship:

$$
\begin{gathered}
H_{\mathrm{tot}}=s_{3} /\left[c_{2} v_{12}+\left(c_{2} v_{22}-c_{2} v_{12}\right) y-s_{3} v_{12} h_{12}\right. \\
\left.-s_{3}\left(v_{22} h_{22}-v_{12} h_{12}\right) y\right] .
\end{gathered}
$$

Now $y=H_{2} /\left(H_{1}+H_{2}\right)$ denotes the fraction of defended herbivores. The line of reasoning is similar to the bitrophic case. At a certain carrying capacity three trophic levels can exist. Carnivores will increase under further enrichment (see for example Fig. 3, top panel), causing the fraction of defended herbivores, $y$, to increase (Fig. 3, middle panel). From Eq. 14 it follows that an increase in $y$ will cause the entire equilibrium herbivore level to increase when $s_{3}\left(v_{22} h_{22}-v_{12} h_{12}\right)>$ $c_{2}\left(v_{22}-v_{12}\right)$. If we assume that $v_{12}=v_{22}$, this inequality simplifies to $s_{3}\left(h_{22}-h_{12}\right)>0$, which is true when $h_{22}$ $>h_{12}$. This implies that the equilibrium herbivore level increases, under enrichment in a tritrophic system, when induced defenses increase handling times.

\section{Numerical results}

When considering the planktonic model system, algae will be referred to as plants, herbivorous rotifers as herbivores, and predatory rotifers as carnivores.

In a bitrophic system the plant level increases gradually when plant defenses are inducible (Fig. 1, bottom panel). This gradual increase occurs for the productivity range in which the fraction of defended plants increases from 0 to almost 1 (Fig. 4, bottom panel). When almost all plants have induced defenses, biomass responses follow EEH predictions, as in the scenarios with no and permanent defenses (Fig. 1). The productivity range for which both defended and undefended plants are present widens as the half-saturation value of defense induction $g_{1}$ (see Eqs. 3a,b) increases (Fig. 4 , bottom panel), and for the fraction of defended plants where the carrying capacity is very large $(k \rightarrow \infty)$ we have $x_{\text {sup }}=0.997,0.9724,0.7525$ for $g_{1}=0.06,0.54$, 1.62 , respectively. Within this productivity range total plant biomass increases gradually (Fig. 4, middle panel).

Equilibrium herbivore biomass increases with enrichment in all bitrophic defense scenarios (Fig. 1, top panel). A seemingly counterintuitive result is that herbivores can attain a higher abundance in the permanent and inducible defense scenarios than in the no-defense

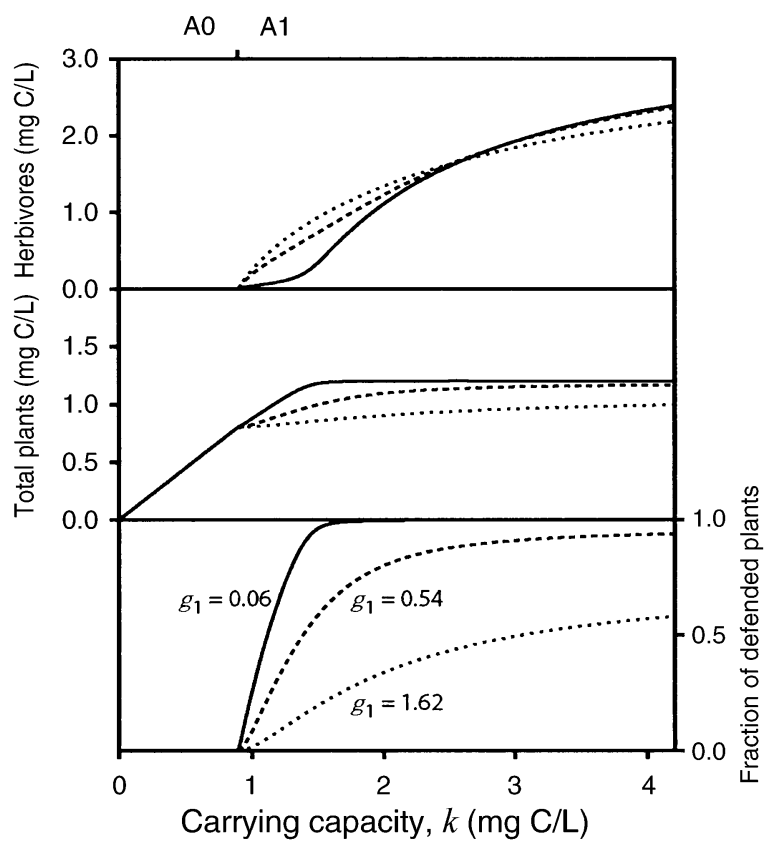

FIG. 4. Effects of inducible defenses on plant-herbivore biomass responses to enrichment. Population densities of herbivores (top), plants (middle), and the fraction of defended plants (bottom), $x=P_{2} /\left(P_{1}+P_{2}\right)$, are shown for different values of $g_{1}$, the consumer density at which half the maximum defense induction rate is reached. The default value of $g_{1}$ is 0.06 . A 0 marks a range of low carrying capacities where only plants are sustained. The area beneath A1 indicates carrying capacities that allow a stable equilibrium of plants and herbivores. 
scenario, at higher values of the carrying capacity (Fig. 1, top panel). This result directly follows from Eq. 7, for $k \rightarrow \infty$. Equilibrium herbivore biomass is higher in the scenario with defended plants than in the scenario with undefended plants, i.e., $\lim _{k \rightarrow \infty} H^{*}$ in the defense scenario is greater than $\lim _{k \rightarrow \infty} H^{*}$ in the no-defense scenario for

$$
\left[\frac{r_{1}-s_{21}}{v_{21}\left(c_{1}-h_{21} s_{12}\right)}\right]>\left[\frac{r_{1}-s_{11}}{v_{11}\left(c_{1}-h_{11} s_{12}\right)}\right]
$$

where $P_{1}^{*}$ from Eq. 6, and analogously $P_{2}^{*}$ have been substituted into Eq. 7. This inequality holds for the planktonic model system under study (see Table 2).

In the tritrophic food chain that has inducible defenses in both plants and herbivores, all trophic levels increase gradually in response to enrichment (Fig. 3). In contrast, equilibrium herbivore biomass remains constant in the no-defense and permanent defense scenarios, thus following EEH predictions (Fig. 3). At the highest levels of primary productivity shown in Fig. 3 , population densities fluctuate in all three defense scenarios. Within this productivity range average carnivore density may decrease under enrichment in the scenario with permanent defenses (Fig. 3, top), as previously noted by Abrams and Roth (1994).

The fraction of defended herbivores increases along the entire productivity gradient for which the tritrophic system is stable. It is interesting to note that not all plants become defended in the tritrophic food chain. Plants have the same low value of $g_{1}$ as in the bitrophic scenario, where almost all plants became defended within a narrow productivity gradient. The difference is caused by carnivores that have a sufficient top-down effect on herbivores to prevent a full induction of plant defenses. A comparison of the bitrophic and tritrophic results in Figs. 1 and 3 shows that inducible defenses do not completely neutralize top-down effects in this model system. There is still a trophic cascade, although the direct effect of the carnivore on herbivore density is much stronger than the indirect effect on plant density.

\section{Sensitivity analysis}

The range of primary productivities that allows both plants and herbivores to increase in biomass is governed by the dependence of defense induction on herbivore density (see Fig. 4, where $g_{1}$ is increased). This range widens when the defense level of induced plants is increased quantitatively. Defense levels were changed in three ways, by increasing handling times, reducing attack rates, and decreasing the conversion efficiency from plants to herbivores. In all these cases an increase in the quantitative level of defenses causes a larger increase in plant biomass in response to enrichment, for a wider range of productivities.

The sensitivity analysis further shows that qualitatively different types of induced defenses, that affect either attack rates, or handling times or conversion ef- ficiencies, have the same effect on biomass responses of plants and herbivores. In all these cases inducible defenses allow an increase in productivity to increase the biomass of adjacent trophic levels. This result is obtained both when defended plants are edible but more difficult to handle, and when they are completely inedible. Induced colonies, spines, or thorns may have either of these effects, depending on herbivore size. Our results are robust to different ways of incorporating trade-offs: productivity increases the biomass of adjacent trophic levels when there are either reduced growth rates or increased mortality rates, or no costs at all to producing a defense. The behavior of the system is insensitive to changes in the value of induction and decay parameter $i_{1}$, as long as this value is high relative to $\left(s_{21}-s_{11}\right)$. This ensures that almost all plants can be in the defended state at high herbivore densities.

\section{Comparison of heterogeneous bitrophic scenarios with and without inducible defenses}

Plant and herbivore biomass both increased under enrichment in the intraspecific heterogeneity scenarios with inducible defenses, where $v_{21}<v_{11}$. This occurred when $h_{21}>h_{11}$, when $h_{21}=h_{11}$, and when $h_{21}=h_{11}=$ 0 . In these cases, defended plant biomass increased more strongly with enrichment than undefended plant biomass decreased. When defended plants are inedible $\left(v_{21}=0\right)$ undefended plant biomass remained constant, while densities of defended plants and herbivores increased.

Results were more variable in the interspecific heterogeneity scenarios with two plant species. When the herbivore had a linear functional response $\left(v_{21}<v_{11}\right.$, $h_{21}=h_{11}=0$ ), undefended plants decreased, defended plants increased, and herbivore biomass remained unchanged. This case is similar to the one in Leibold (1996; Fig. 7). These biomass responses also occurred when the herbivore had a Holling type II functional response and identical handling times on defended and undefended plants $\left(v_{21}<v_{11}\right.$ and $\left.h_{21}=h_{11}\right)$. However, when handling times were larger on defended plants $\left(v_{21}<v_{11}\right.$ and $\left.h_{21}>h_{11}\right)$, undefended plants decreased, while defended plants, herbivores, and total plant biomass increased. In the special case where defended plants were inedible $\left(v_{21}=0\right)$, only defended plant biomass increased, with undefended plants and herbivores remaining unaffected. These results for $\left(v_{21}=0\right)$ are similar to those in Phillips (1974), Leibold (1996), and Bohannan and Lenski (1999). Kretzschmar et al. (1993) obtained a different result for this case, due to a simplifying assumption (see Discussion).

\section{Discussion}

Current theory on trophic structure recognizes the importance of resource edibility in determining the relative magnitude of bottom-up and top-down control. Much work has focused on the effects of inedible species and has assumed that defenses are a fixed trait. 
However, in natural food webs many plant and herbivore species have inducible defenses. Such defenses are flexible, respond to consumer densities, and cause intraspecific heterogeneity. Both defended and undefended individuals may be present at a certain density of resources and consumers.

Here we show that such intraspecific heterogeneity causes a gradual increase in the biomass of all trophic levels under enrichment. This pattern is in agreement with data from a variety of laboratory and field studies and in contrast with predictions of the classical Oksanen et al. (1981) model, that assumes complete topdown control of plants in bitrophic systems and of herbivores in tritrophic systems.

The mechanism that allows inducible defenses to increase the relative importance of bottom-up control is explained mathematically in the Results section and illustrated in ecological terms in the following bitrophic scenario. An increase in the primary productivity of plants necessarily causes an increase in the herbivore level (given that all plants are edible to some extent). This increase in herbivore density will cause defenses to be induced and part of the plant level to be in the defended state. This decreases plant edibility, lowers the per capita assimilation rate by herbivores, and results in an increase of total plant biomass. Thus both trophic levels increase under enrichment. This can be seen by considering the herbivore at equilibrium: here the herbivore's mortality balances its growth, which is simply its per capita assimilation rate of plants multiplied by plant density. Since the herbivore's mortality rate is a constant, any decrease in the per capita assimilation rate of the herbivore has to be compensated by a higher plant biomass at equilibrium. This increase in the entire plant level occurs as long as the fraction of defended plants increases with herbivore density under enrichment (Eqs. 9, 10). The range of carrying capacities for which this condition holds depends on the herbivore density $g_{1}$ at which plant defense induction reaches half its maximum rate. For large values of $g_{1}$ the fraction of defended plants increases for a wide range of carrying capacities, as illustrated in Fig. 4. How natural selection will act on $g_{1}$ will depend on the costs and benefits of induced defenses. We expect larger values of $g_{1}$ in systems where the costs of induced defenses are relatively high.

\section{Interspecific vs. intraspecific heterogeneity}

A bitrophic food chain with homogeneous trophic levels and fixed (permanent) defenses contrasts with the above scenario in that the plant level does not change under enrichment. Since inducible defenses (intraspecific heterogeneity) and the presence of different species (intraspecific heterogeneity) both have withintrophic-level heterogeneity as a result, a comparison of the two is warranted.

Abrams (1993) showed that trophic level responses may differ from the Oksanen et al. (1981) predictions when different species or types are present within one or more trophic levels. Part of these alternative responses was due to the fact that the analyses allowed differential inputs to the growth of different resource species. This is not the case in the present study, where the different intraspecific plant types, one with induced defenses and the other undefended, are always enriched by the same factor.

The interspecific heterogeneity model produces two qualitatively different trophic biomass responses to enrichment, (1) equilibrium densities of both plants and herbivores increase gradually, and (2) equilibrium densities of plants increase while herbivores remain unchanged. The first pattern occurs when herbivores have a Holling type II functional response and $\left(0<v_{21}<\right.$ $v_{11}$ and $\left.h_{21}>h_{11}\right)$. The second pattern occurs when (a) $\left(0<v_{21}<v_{11}\right.$ and $\left.h_{21}=h_{11} \geq 0\right)$, and (b) $\left(v_{21}=0\right)$. The latter case (2b) has been discussed in Phillips (1974), Grover (1994), Leibold (1996), and Bohannan and Lenski (1999). Kretzschmar et al. (1993) also considered this case without a direct interaction between herbivores and inedible plants. These authors derived the result that undefended plant density does not change, while defended plants increase and herbivores increase (Kretzschmar et al. 1993:54). This result for herbivores is peculiar and probably results from their simplifying assumption that the edible plant has no effect (whatsoever) on the inedible plant's growth. Without this unrealistic simplification the Kretzschmar et al. (1993) model produces the result mentioned above as case (2b).

Predictions of the inducible defense model (with intraspecific heterogeneity) are robust to any of the above changes $(1,2 \mathrm{a}, \mathrm{b})$ : Equilibrium plant and herbivore biomass are both predicted to increase in response to increased primary productivity. This increase in adjacent trophic levels is contingent on an increase in the fraction of defended plants along the productivity gradient, as explained above. The predictions made by the inducible defense model are in agreement with several studies that have shown the biomass of adjacent trophic levels to increase with primary productivity (Akcakaya et al. 1995, Leibold 1996, Brett and Goldman 1997, Kaunzinger and Morin 1998). This pattern may also occur in the case of interspecific heterogeneity (scenario 1), but this multispecies model predicted the herbivore level to remain constant in scenarios (2a and $2 b$ ), which is not consistent with the above empirical data.

\section{Analytical and numerical results}

The analytical results (see Eqs. 9-13) on biomass responses in a bitrophic system are general, but depend on the assumption that inducible defenses only affect handling times. The numerical work and sensitivity analysis show that the same results may apply when inducible defenses affect attack rates or conversion efficiencies. Numerical results for bitrophic and tritroph- 
ic systems indicate that inducible defenses cause the biomass of all trophic levels to gradually increase in response to enrichment. This result was obtained when defense induction resulted in intraspecific heterogeneity of the prey level. Such heterogeneity occurs for a wider range of productivities when defenses have a strong quantitative effect and when induction saturates at higher consumer densities.

\section{Toward a multicausal explanation of trophic structure}

We do not claim that inducible defenses are the only possible explanation for increases in the biomass of adjacent trophic levels, as even simple patterns are likely to have multiple causes in ecology. Future studies may attempt an integrated explanation of biomass responses and show the relative importance of different ecological factors like ontogenetic niche shifts (Mittelbach et al. 1988), changes in community composition (Leibold 1996, Leibold et al. 1997, Chase et al. 2000), spatial refuge use (Jansen 2001), density dependence (Mittelbach et al. 1988, Abrams 1993, McCann et al. 1998), adaptive foraging by herbivores (Abrams 1984, 1991, 1992, 1993) and inducible defenses. More studies are needed that analyze the interactive effects of potentially important factors. For example, Abrams and Vos (2003) analyzed the effects of middle-level adaptation and density dependence at all trophic levels in a full factorial design. Different mechanisms of adaptive change, such as microevolution, adaptive behavior, species replacement, and induced defenses, were shown to have similar effects on trophic-level responses to a perturbation in the form of increased mortality (Abrams and Vos 2003).

Another approach that may help to unravel the mechanisms that govern trophic structure is an experimental one. This allows manipulation of the above factors by assembling simple communities of species with distinct characteristics. In an intriguing experimental study Kaunzinger and Morin (1998) observed that productivity increased the biomass of all trophic levels, both in bitrophic and tritrophic microbial food chains. This surprising response remained unexplained in that paper, given that much care was taken to exclude several potential sources of heterogeneity. We hypothesize that consumer-induced morphological changes may have caused these results, that are in agreement with our model predictions. The results presented do not allow us to directly test this hypothesis, since morphological changes were not monitored in this experiment, which used Serratia marcescens as bacterial basal prey and Colpidium striatum ciliates as intermediate prey of the predatory ciliate Didinium nasutum. Indirect evidence suggests a possible role of induced morphological changes. First, Serratia marcescens is well known for the existence of different phenotypes, including elongated individuals that may exhibit coordinated multicellular swarming behavior through quorum sensing
(Ang et al. 2001). Secondly, several species in the Colpidium genus are known to exhibit predator-induced morphological changes (Fyda 1998, Kuhlmann et al. 1999). Holyoak noted an increase in C. striatum size in the presence of its predator $D$. nasutum, but the relative roles of predator-released infochemicals and increased per capita food availability for C. striatum could not be determined (M. Holyoak, personal communication).

Our results show that intraspecific heterogeneity resulting from consumer-induced defenses causes adjacent trophic levels to increase in response to enrichment, thus enhancing the propagation of bottom-up effects. This makes such intraspecific heterogeneity a candidate component of a more complete explanation of biomass responses in nature.

\section{ACKNOWLEDGMENTS}

We are grateful to Koos Vijverberg, Kevin McCann, and two referees for perceptive comments and useful suggestions. M. Vos thanks Peter Abrams for insightful remarks on the costs of inducible defenses. We thank Christina Kaunzinger, Marcel Holyoak, Janusz Fyda, Dalius Balciunas, Sharon Lawler and Lin Jiang for answering our questions on the Colpidium-Didinium system. M. Vos was supported by contract ENV4-CT97-0402 within the framework of the European Commission's Environment and Climate Programme, which is part of the project network WAtER (Wetland and Aquatic Ecosystem Research). This is publication 3340 of the Netherlands Institute of Ecology (NIOO-KNAW).

\section{Literature Cited}

Abrams, P. A. 1984. Foraging time optimization and interactions in food webs. American Naturalist 124:80-96.

Abrams, P. A. 1991. Strengths of indirect effects generated by optimal foraging. Oikos 62:167-176.

Abrams, P. A. 1992. Predators that benefit prey and prey that harm predators: unusual effects of interacting foraging adaptations. American Naturalist 140:573-600.

Abrams, P. A. 1993. Effect of increased productivity on the abundance of trophic levels. American Naturalist 141:351371 .

Abrams, P. A., and J. D. Roth. 1994. The effects of enrichment of three-species food chains with nonlinear functional responses. Ecology 75:1118-1130.

Abrams, P. A., and M. Vos. 2003. Adaptation, density dependence and the responses of trophic level abundances to mortality. Evolutionary Ecology Research 5:1113-1132.

Agrawal, A. A. 2001. Phenotypic plasticity in the interactions and evolution of species. Science 294:321-326.

Akcakaya, H. R., R. Arditi, and L. R. Ginzburg. 1995. Ratiodependent predation: an abstraction that works. Ecology 76:995-1004.

Ang, S., Y. T. Horng, J. C. Shu, P. C. Soo, J. H. Liu, W. C. Yi, H. C. Lai, K. T. Luh, S. W. Ho, and S. Swift. 2001. The role of RsmA in the regulation of swarming motility in Serratia marcescens. Journal of Biomedical Science 8: 160-169.

Anholt, B. R., and E. E. Werner. 1999. Density-dependent consequences of induced behavior. Pages 218-230 in R. Tollrian and C. D. Harvell, editors. The ecology and evolution of inducible defenses. Princeton University Press, Princeton, New Jersey, USA.

Bohannan, B. J. M., and R. E. Lenski. 1999. Effect of prey heterogeneity on the response of a model food chain to resource enrichment. American Naturalist 153:73-82. 
Brett, M. T., and C. R. Goldman. 1997. Consumer versus resource control in freshwater pelagic food webs. Science 275:384-386.

Brönmark, C., L. B. Petterson, and P. A. Nilsson. 1999. Predator-induced defense in crucian carp. Pages 213-217 in R. Tollrian and C. D. Harvell, editors. The ecology and evolution of inducible defenses. Princeton University Press, Princeton, New Jersey, USA.

Chase, J. M., M. A. Leibold, A. L. Downing, and J. B. Shurin. 2000. The effects of productivity, herbivory, and plant species turnover in grassland food webs. Ecology 81:24852497.

Dahl, J., and B. L. Peckarsky. 2002. Induced morphological defenses in the wild: predator effects on a mayfly, Drunella coloradensis. Ecology 83:1620-1634.

DeMeester, L., P. Dawidowicz, E. van Gool, and C. J. Loose. 1999. Ecology and evolution of predator-induced behavior of zooplankton: depth selection behavior and diel vertical migration. Pages 160-176 in R. Tollrian and C. D. Harvell, editors. The ecology and evolution of inducible defenses. Princeton University Press, Princeton, New Jersey, USA.

Dumont, H. J., I. Van de Velde, and S. Dumont. 1975. The dry weight estimate of biomass in a selection of Cladocera, Copepoda and Rotifera from the plankton, periphyton and benthos of continental waters. Oecologia 19:75-97.

Fussmann, G. F., S. P. Ellner, K. W. Shertzer, and N. G. Hairston, Jr. 2000. Crossing the Hopf bifurcation in a live predator-prey system. Science 290:1358-1360.

Fyda, J. 1998. Predator-induced morphological changes in the ciliate Colpidium (Protozoa, Ciliophora). European Journal of Protistology 34:111-117.

Gilbert, J. J. 1967. Asplanchna and postero-lateral spine production in Brachionus calyciflorus. Archiv für Hydrobiologie 64:1-62.

Gilbert, J. J. 1976. Polymorphism in the rotifer Asplanchna sieboldii: biomass, growth, and reproductive rate of the saccate and campanulate morphotypes. Ecology 57:542551

Gilbert, J. J. 1999. Kairomone-induced morphological defences in rotifers. Pages 127-141 in R. Tollrian and C. D. Harvell, editors. The ecology and evolution of inducible defenses. Princeton University Press, Princeton, New Jersey, USA.

Gilbert, J. J., and J. K. Waage. 1967. Asplanchna, Asplanchna-substance and posterolateral spine length variation of the rotifer Brachionus calyciflorus in a natural environment Ecology 48:1027-1031.

Gomez, J. M., and R. Zamora. 2002. Thorns as induced mechanical defense in a long-lived shrub (Hormathophylla spinosa, Cruciferae). Ecology 84:885-890.

Gowda, J. H. 1996. Spines of Acacia tortilis: what do they defend and how? Oikos 77:279-284.

Grover, J. P. 1994. Assembly rules for communities of nutrient-limited plants and specialist herbivores. American Naturalist 143:258-282.

Grover, J. P. 1995. Competition, herbivory, and enrichment: nutrient-based models for edible and inedible plants. American Naturalist 145:746-774.

Hansson, L. A. 1992. The role of food chain composition and nutrient availability in shaping algal biomass development. Ecology 73:241-247.

Harvell, C. D. 1999. Complex biotic environments, coloniality and heritable variation for inducible defenses. Pages 231-244 in R. Tollrian and C. D. Harvell, editors. The ecology and evolution of inducible defenses. Princeton University Press, Princeton, New Jersey, USA.

Havel, J. E. 1987. Predator-induced defenses: a review. Pages 263-278 in W. C. Kerfoot and A. Sih, editors. Predation direct and indirect impacts on aquatic communities, Uni- versity Press of New England, Hanover, New Hampshire, USA.

Hessen, D. O., and E. Van Donk. 1993. Morphological changes in Scenedesmus induced by substances released from Daphnia. Archiv fur Hydrobiologie 127:129-140.

Iyer, N., and T. R. Rao. 1996. Responses of the predatory rotifer Asplanchna intermedia to prey species differing in vulnerability: laboratory and field studies. Freshwater Biology 36:521-533.

Jansen, V. A. A. 2001. The dynamics of two diffusively coupled predator-prey populations. Theoretical Population Biology 59:119-131.

Jeschke, J. M., and R. Tollrian. 2000. Density-dependent effects of prey defences. Oecologia 123:391-396.

Jørgensen, S. E., S. N. Nielsen, and L. A. Jørgensen. 1991. Handbook of ecological parameters and ecotoxicology. Elsevier Science, Amsterdam, The Netherlands.

Karban, R., and I. T. Baldwin. 1997. Induced responses to herbivory. University of Chicago Press, Chicago, Illinois, USA.

Kaunzinger, C. M. K., and P. J. Morin. 1998. Productivity controls food chain properties in microbial communities. Nature 395:495-497.

Kretzschmar, K., R. M. Nisbet, and E. McCauley. 1993. A predator-prey model for zooplankton grazing on competing algal populations. Theoretical Population Biology 44:3266

Kuhlmann, H. W., J. Kusch, and K. Heckmann. 1999. Predator-induced defenses in ciliated protozoa. Pages 142-159 in R. Tollrian and C. D. Harvell, editors. The ecology and evolution of inducible defenses. Princeton University Press, Princeton, New Jersey, USA.

Lampert, W., K. O. Rothhaupt, and E. von Elert. 1994. Chemical induction of colony formation in a green alga (Scenedesmus acutus) by grazers (Daphnia). Limnology and Oceanography 39:1543-1550.

Larocque, I., A. A. Mazumder, M. Proulx, D. R. S. Lean, and F. R. A. Pick. 1996. Sedimentation of algae: relationships with biomass and size distribution. Canadian Journal of Fisheries and Aquatic Science 53:1133-1142.

Leibold, M. A. 1989. Resource edibility and the effects of predators and productivity on the outcome of trophic interactions. American Naturalist 134:922-949.

Leibold, M. A. 1996. A graphical model of keystone predators in food webs: trophic regulation of abundance, incidence, and diversity patterns in communities. American Naturalist 147:784-812.

Leibold, M. A., J. M. Chase, J. B. Shurin, and A. L. Downing. 1997. Species turnover and the regulation of trophic structure. Annual Review of Ecology and Systematics 28:467494.

Lively, C. M. 1999. Developmental strategies in spatially variable environments: barnacle shell dimorphism and strategic models of selection. Pages 245-258 in R. Tollrian and C. D. Harvell, editors. The ecology and evolution of inducible defenses. Princeton University Press, Princeton, New Jersey, USA.

Lürling, M. 1999. The smell of water. Grazer-induced colony formation in Scenedesmus. Dissertation. Wageningen University, Wageningen, The Netherlands.

Lürling, M., and E. Van Donk. 2000. Grazer-induced colony formation in Scenedesmus: are there costs to being colonial? Oikos 88:111-118.

McCann, K. S., A. Hastings, and D. R. Strong. 1998. Trophic cascades and trophic trickles in pelagic food webs. Proceedings of the Royal Society of London B 265:205-209.

Milchunas, D. G., and W. K. Lauenroth. 1993. Quantitative effects of grazing on vegetation and soils over a global range of environments. Ecological Monographs 63:327366. 
Mittelbach, G. G., C. Osenberg, and M. A. Leibold. 1988. Trophic relations and ontogenetic niche shifts in aquatic ecosystems. Pages 217-235 in B. Ebenman and L. Persson, editors. Size-structured populations: ecology and evolution. Springer, Berlin, Germany.

Nandini, S., and S. S. S. Sarma. 1999. Effect of starvation time on the prey capture behaviour, functional response and population growth of Asplanchna sieboldi (Rotifera). Freshwater Biology 42:121-130.

Oksanen, L., S. D. Fretwell, J. Arruda, and P. Niemela. 1981. Exploitation of ecosystems in gradients of primary productivity. American Naturalist 118:240-261.

Oksanen, L., and T. Oksanen. 2000. The logic and realism of the hypothesis of exploitation ecosystems. American Naturalist 155:703-723.

Phillips, O. M. 1974. The equilibrium and stability of simple marine biological systems. II. Herbivores. Archiv für Hydrobiologie 73:310-333.

Power, M. 1992. Top-down and bottom-up forces in food webs: do plants have primacy? Ecology 73:733-746.

Rosenzweig, M. L. 1968. Net primary productivity of terrestrial communities: predictions from climatological data. American Naturalist 102:67-74.

Rosenzweig, M. L. 1971. Paradox of enrichment: destabilization of exploitation ecosystems in ecological time. Science 171:385-387.

Rosenzweig, M. L. 1973. Exploitation in three trophic levels. American Naturalist 107:275-294.

Rosenzweig, M. L., and R. MacArthur. 1963. Graphical representation and stability conditions of predator-prey interactions. American Naturalist 97:209-223.

Steiner, C. F. 2001. The effects of heterogeneity and consumer identity on the limitation of trophic-level biomass. Ecology 82:2495-2506.
Stemberger, R. S. 1990. Food limitation, spination, and reproduction in Brachionus calyciflorus. Limnology and Oceanography 35:33-44.

Tang, K. W. 2003. Grazing and colony size development in Phaeocystis globosa (Prymnesiophyceae): the role of a chemical signal. Journal of Plankton Research 25:831-842.

Tollrian, R., and S. I. Dodson. 1999. Inducible defenses in cladocera: constraints, costs, and multipredator environments. Pages 177-202 in R. Tollrian and C. D. Harvell, editors. The ecology and evolution of inducible defenses. Princeton University Press, Princeton, New Jersey, USA.

Turner, A. M., R. J. Bernot, and C. M. Boes. 2000. Chemical cues modify species interactions: the ecological consequences of predator avoidance by freshwater snails. Oikos 88: $148-158$.

Van Donk, E., M. Lürling, and W. Lampert. 1999. Consumerinduced changes in phytoplankton: inducibility, costs, benefits, and the impact on grazers. Pages 89-103 in R. Tollrian and C. D. Harvell, editors. The ecology and evolution of inducible defenses. Princeton University Press, Princeton, New Jersey, USA.

Vos, M., B. J. G. Flik, J. Vijverberg, J. Ringelberg, and W. M. Mooij. 2002. From inducible defences to population dynamics: modelling refuge use and life history changes in Daphnia. Oikos 99:386-396.

Walz, N., editor. 1993. Plankton regulation dynamics. Ecological Studies 98. Springer-Verlag, New York, New York, USA.

Wootton, J. T., and M. E. Power. 1993. Productivity, consumers, and the structure of a river food-chain. Proceedings of the National Academy of Sciences (USA) 90:13841387 .

\section{APPENDIX}

Partial derivatives with respect to $k$ for the positive and negative branches of Eq. 13 are available in ESA's Electronic Data Archive: Ecological Archives E085-089-A1. 